\title{
Factors affecting intraoperative and postoperative complications of RIRS classified by the Clavien and Satava grading systems
}

\author{
Omer Koras ${ }^{1}$, Ibrahim Halil Bozkurt ${ }^{2}$, Nihat Karakoyunlu ${ }^{3}$, Serdar Çelik ${ }^{4}$, Ertugrul Sefik ${ }^{5}$, \\ Tansu Degirmenci ${ }^{5}$, Serkan Yarımoğlu ${ }^{4}$, and Salih Polat ${ }^{2}$ \\ ${ }^{1}$ Mustafa Kemal University Faculty of Medicine \\ ${ }^{2}$ Affiliation not available \\ ${ }^{3}$ Ankara Diskapi Yildirim Beyzat Training and Research Hospital \\ ${ }^{4}$ Izmir Bozyaka Training and Research Hospital \\ ${ }^{5}$ University of Health Sciences Izmir Bozyaka Education and Research Hospital
}

March 16, 2021

\begin{abstract}
Objective: To classify intraoperative and postoperative complications using the modified Clavien classification system (MCCS) and modified Satava classification system (SCS) and to evaluate the parameters associated with complications in patients undergoing retrograde intrarenal surgery (RIRS) for renal and proximal ureteral stones. Materials and Methods: We performed a retrospective analysis of 949 patients who underwent RIRS for renal stones and upper ureteral stones at two institutions between March2015 and June2020. Intraoperative complications were assessed using SCC and postoperative complications were graded according to MCCS. Univariate and multivariate analyses were undertaken to determine predictive factors affecting complication rates. Results: The female/male ratio of 949 patients was found to be $346(36.5) / 603(63.5)$. The median stone size was determined as $13 \mathrm{~mm}$. The stone-free rate was $83.6 \%$ after the first intervention, and the final stone-free rate was $94.4 \%$ after re-procedure. According to SCS, the intraoperative event and complication incidence was 153(16.1\%). MCCS revealed postoperative complications in $121(12.8 \%)$ patients. Major complications were observed in $18(1.9 \%)$ patients. The rate of complications was higher in patients with renal anomalies $(9.9 \%$ vs $3.9 \%, \mathrm{p}=0.006)$. Besides, stone localization, size, number and density were associated with the development of complications $(\mathrm{p}<0.001, \mathrm{p}<0.001, \mathrm{p}<0.001$ and $\mathrm{p}=0.002$, respectively). In addition, the multivariate analysis revealed that for the patients with grade[?]3 complications according to MCCS, only stonefree status was a significant predictor of complication development $(\mathrm{p}=0.044)$ whereas for those with grade [?]2b complications according to SCS, significant predictors were stone size $(\mathrm{p}<0.001)$, stone density $(\mathrm{p}=0.022)$, and fluoroscopy time ( $<<0.001)$. Conclusion: This study showed that abnormal kidney anatomy, multiple stones, operative time, and stone-free status were reliable predictors of complication development during and after RIRS. Appropriate preoperative management should be planned according to these predictors to prevent intraoperative and postoperative complications.
\end{abstract}

Factors affecting intraoperative and postoperative complications of RIRS classified by the Clavien and Satava grading systems

\begin{abstract}
Objective: To classify intraoperative and postoperative complications using the modified Clavien classification system (MCCS) and modified Satava classification system (SCS) and to evaluate the parameters associated with complications in patients undergoing retrograde intrarenal surgery (RIRS) for renal and proximal ureteral stones.
\end{abstract}

Materials and Methods: We performed a retrospective analysis of 949 patients who underwent RIRS for renal stones and upper ureteral stones at two institutions between March2015 and June2020. Intraoperative 
complications were assessed using SCC and postoperative complications were graded according to MCCS. Univariate and multivariate analyses were undertaken to determine predictive factors affecting complication rates.

Results: The female/male ratio of 949 patients was found to be $346(36.5) / 603(63.5)$. The median stone size was determined as $13 \mathrm{~mm}$. The stone-free rate was $83.6 \%$ after the first intervention, and the final stone-free rate was $94.4 \%$ after re-procedure. According to SCS, the intraoperative event and complication incidence was $153(16.1 \%)$. MCCS revealed postoperative complications in 121(12.8\%) patients. Major complications were observed in 18(1.9\%) patients. The rate of complications was higher in patients with renal anomalies $(9.9 \%$ vs $3.9 \%, p=0.006)$. Besides, stone localization, size, number and density were associated with the development of complications ( $p<0.001, p<0.001, p<0.001$ and $p=0.002$, respectively). In addition, the multivariate analysis revealed that for the patients with grade[?]3 complications according to MCCS, only stone-free status was a significant predictor of complication development $(p=0.044)$ whereas for those with grade [?]2b complications according to SCS, significant predictors were stone size $(p<0.001)$, stone density $(p=0.022)$, and fluoroscopy time $(p<0.001)$.

Conclusion: This study showed that abnormal kidney anatomy, multiple stones, operative time, and stone-free status were reliable predictors of complication development during and after RIRS. Appropriate preoperative management should be planned according to these predictors to prevent intraoperative and postoperative complications.

Keywords: Retrograde intrarenal surgery, complication, Clavien, Satava, renal stone

\section{What's already known about this topic?}

Although the literature contains research reporting retrograde intrarenal surgery complications, only a limited number of studies have investigated both intraoperative and postoperative complications according to Clavien and Satava classifications in large series.

\section{What does this article add?}

This study reviewed and reclassified the complications during or after retrograde intrarenal surgery in light of the literature. It is also one of the first studies in the literature to classify complications according to the Clavien and Satava systems. Furthermore, it reveals situations that may require additional intervention or secondary operation as pre-operative predictive factors.

Acknowledgements: None

Funding: None

Conflict of interest: The authors declare that they have no conflict of interest.

Word count of text: 3016

\section{Word count of Abstract: 296}

\section{Introduction}

Since retrograde intrarenal surgery (RIRS) was first defined in the 1990s, there have been improvements concerning this technique [1,2]. With the development of flexible endoscopes (miniaturization of endoscopes and improvement in image quality), increase in the quality of light sources, and advances in the lithotripsy technology, RIRS has become the first treatment option for upper urinary tract stones of $<2 \mathrm{~cm}[3,4]$. In parallel to these developments, the treatment of proximal ureteral and renal stones has undergone a significant change in recent years. In a systemic review, the total stone-free rate was reported as $91 \%$ and the major complication rate (Clavien [?]3) as $4.5 \%$ in an average of 1.45 procedures in patients who underwent RIRS due to having renal stones of $>2 \mathrm{~cm}[5,6]$. Although there are large series in the literature investigating 
the complications of semirigid ureteroscopy [7,8], only a limited number of studies have been conducted to evaluate RIRS complications in large series. Some scoring systems have been defined for grading surgical complications. Among these, the modified Clavien classification system (MCCS) is most commonly used for postoperative complications, and the modified Satava classification system (SCS) is employed to classify intraoperative complications and events $[9,10]$. Complication classification systems are important because they can easily demonstrate the safety of the surgery and also create standardization to compare results obtained from different centers. In addition, determining parameters associated with complications and predicting complication development are as important as achieving stone-free status, which is considered to indicate the success of surgery.

In this study, we aimed to classify intraoperative and postoperative complications in patients undergoing RIRS using MCCS and SCS and to evaluate complication-related parameters.

\section{Materials and Methods}

The data of 949 patients, who underwent RIRS for renal and proximal ureteral stones at HSU Izmir Bozyaka Training and Research Hospital and HSU Ankara Diskapi Training and Research Hospital between March 2015 and June 2020, were retrospectively analyzed. Patient data obtained included age, sex, body mass index (BMI), history and physical examination findings, specific comorbidities, and the American Society of Anesthesiologists (ASA) physical status class risk. All patients were evaluated with a detailed history before the operation. Stone parameters, namely number, diameter, location and CT attenuation value of the stone, previous treatments applied for the stone, operative time, fluoroscopy time, and stone burden (length $\mathrm{x}$ width $\mathrm{x} \pi \mathrm{x} 0.25)$ were recorded. For multiple stones, the sum of each stone's volume was used. The patients were evaluated preoperatively with complete blood count, serum creatinine, bleeding and clotting times, and urine culture analyses. Those with growth in urine cultures were operated on after antibiotherapy was administered for a sufficient time and the control urine cultures were sterile. Patients with missing data and/or those that did not undergo post-operative first-month non-contrast CT were excluded from the study. Patients who underwent diagnostic ureteroscopy but did not have related data were also not included in the study. Ethics approval was obtained from the Ethics Committee.

All operations were performed under general anesthesia in the dorsal lithotomy position. First, ureteroscopy was performed with a semirigid ureteroscope to provide mechanical dilatation and place a guide wire. Then, according to the surgeon's preference, a ureteral access sheath (UAS) ( Flexor 9.5/11.5Fr or 12/14Fr, Cook Medical Bloomington, IL, USA, Navigator 11/13Fr, Boston Scientific, Natik, MA, USA) was placed over the guide wire under fluoroscopic control. In cases whereas UAS could not be placed, the operation was performed with a sheath. If the flexible ureteroscope could not reach the kidney over the guide wire, a double-J (DJ) stent was placed and left for passive dilatation, and the operation was postponed. All the RIRS procedures were performed using flexible ureteroscopes (Flex-X2, Karl Storz Endoscope, Tuttligen, Germany), and the stones were fragmented with a Holmium laser using a 6-14 W range. If needed, some fragments were removed with tipless nitinol stone baskets. The procedure was terminated after stone-free status was confirmed by both ureteroscopic inspection and fluoroscopy (leaving only ungraspable gravel or fragments $<2 \mathrm{~mm}$ ) or if the surgeon decided to terminate the surgery due to complications, such as bleeding. At the end of the operation, a DJ stent was placed according to the surgeon's preference.

All patients were evaluated with non-contrast CT at the first month after RIRS to evaluate stone-free status. Residual fragments of $<2 \mathrm{~mm}$ were accepted as insignificant. The results were compared in terms of the predictive capability of stone-free status and complications. Intraoperative complications were assessed using SCC, and postoperative complications were graded according to MCCS $[9,10]$.

\section{Statistical analysis}

Categorical data were presented as numbers and percentages. The Shapiro-Wilk test was used to determine whether the distributions of continuous variables were normal. Normally distributed continuous data were presented as mean and standard deviation, and non-normally distributed continuous data as median and interquartile range values. The groups of normally distributed data were compared with the independent 
t-test, and the Mann-Whitney U test was used to compare non-normally distributed data.

The frequencies of categorical variables were compared using the Pearson chi-Square, Yates' chi-square or Fisher's exact test where appropriate. Statistical significance was considered when the p value was $<0.05$. The Bonferroni correction was used in the pairwise comparison of more than two groups with statistically significant differences. The multivariate logistic regression analysis was performed to determine the predictors of complication. All statistical analyses was performed using the Statistical Package for the Social Sciences version 21 (IBM SPSS Statistics; IBM Corp., Armonk, NY).

\section{Results}

The characteristics of the patients and stones are shown in Table 1 . The mean age of the patients was 47.2 years, and the female/male ratio was $346(36.5 \%) / 603(63.5 \%)$. Of all patients, $87.9 \%$ had an ASA score of I and II, 104 had ASA III and 11 had ASA IV. The median stone size was determined as $13 \mathrm{~mm}$. The stone size was $10 \mathrm{~mm}$ or below in $38.9 \%$ of the patients, $11-20 \mathrm{~mm}$ in $41.4 \%$, and larger than $20 \mathrm{~mm}$ in $19.7 \%$. Two or more stones were present in $297(31.3 \%)$ of the patients. The most frequent primary location of the stones was the pelvis $(23.1 \%)$, followed by the lower calyx $(20.7 \%)$, proximal ureter $(18.5 \%)$, multicaliceal $(13.9 \%)$, middle calyx $(8.2 \%)$, both the kidney and ureter $(7.5 \%)$, and upper calyx $(5.0 \%)$.

The perioperative and postoperative data are presented in Table 1. The median operative time and fluoroscopy time were $45 \mathrm{~min}$ and $20 \mathrm{sec}$, respectively. UAS were used in $76 \%$ of the patients and postoperative stents in $92.6 \%$. While the stone-free rate was $83.6 \%$ after the first intervention, the final stone-free rate was $94.4 \%$ after re-procedure.

According to SCS, the incidence of intraoperative event and complications were 153 (16.1\%). The classification of these complications according to the SCS system is shown in Table 2. There were $6.3 \%$ grade 1 , $2.7 \%$ grade $2 \mathrm{a}$ and $7.1 \%$ grade $2 \mathrm{~b}$ complications. While mild bleeding $(2.0 \%)$ and minimal mucosal injury $(3.7 \%)$ were the most common grade 1 complications, recurrent RIRS requirement (5.6\%) after inability to reach the stone due to various reasons was recorded as the most common grade $2 \mathrm{~b}$ complication. Grade [?]2b complications according to SCS, significant predictors were stone size (OR:1.066, 95\% CI =1.036-1.098) $(p$ $<0.001$ ), stone density (OR:0.999, 95\% CI =0.997-1.000) $(p=0.022)$, and fluoroscopy time (OR:1.007, 95\% $\mathrm{CI}=1.003-1.011)(p<0.001)$.

Postoperative complications occurred in 121 (12.8\%) patients. The classification of complications according to MCCS is presented in Table 2. Minor complications (grade 1 and 2) developed in $10.8 \%$ of the patients. Major complications were observed in 18 (1.9\%) patients. Ureteral stricture $(0.2 \%)$ developed in two patients. In addition, two of three patients that developed urosepsis died as a result of multi-organ failure.

Table 3 shows the comparison of possible factors related to complications. The mean age was found to be significantly higher in patients with complications. Complications were higher in patients with renal anomalies $(9.9 \%$ vs $3.9 \%, p=0.006)$. Stone localization was also associated with the development of complications. In the subgroup analysis of stone localization, lower complication rates were detected for the stones located in the pelvis $(14.9 \%$ vs $24.9 \%)$ and proximal ureter $(10.7 \%$ vs $19.7 \%)$ while multicaliceal $(24 \% 12.4 \%)$ and pelvic + single caliceal $(7.4 \%$ vs $2.5 \%)$ stones had significantly higher complication rates. Stone size, number and density were determined to be associated with the development of complications $(p$ $<0.001, p<0.001$ and $p=0.002$, respectively).

Table 4 presents the results of the multivariate analysis of the parameters determined to be associated with the development of complications. Abnormal renal anatomy, multiple stones, operative time, and stone-free status were determined as the independent indicators of complication development. Complication development was increased by 2.7 times by abnormal renal anatomy and presence of multiple stones while it was decreased by 4.2 times by stone-free status ( $p=0.010, p<0.001$, and $p<0.001$, respectively). The multivariate analysis revealed that for the patients with grade [?]3 complications according to MCCS, only stone-free status was a significant predictor of complication development [odds ratio (OR):0.347,95\% CI=0.124-0.974] $(p=0.044)$. 


\section{Discussion}

Renal and proximal ureteral stone disease is a common condition worldwide. The lifetime risk of urolithiasis is $13 \%$ in men and $7 \%$ in women [11]. In recent years, RIRS is widely used in surgical procedures in urology, as in other surgical departments, due to the tendency toward minimally invasive options. RIRS has been compared with other therapeutic modalities in terms of stone-free status and complications $[12,13]$. The stone-free rate of this surgical procedure in our series was $83.6 \%$, and the final stone-free rate after re-procedure was $94.4 \%$. In the literature, the stone-free rate is reported to range from 69.7 to $97 \%$ [1416]. Although minor complications are common after RIRS, major complications such as severe bleeding and sepsis may also develop [17]. Breda et al. reported complications in $8 \%$ of the patients after RIRS, and major complications were present in 1.9\% [18]. SCS and MCCS are the most widely used surgical complication classification systems in the urology discipline $[9,10,19]$. Although there are a few studies reporting complications related to the use of flexible ureteroscopy, only limited research has attempted to standardize complications using these new classification systems. In our study, the incidence of intraoperative events or complications was $153(16.1 \%)$ according to SCC and the incidence of postoperative complications was $121(12.6 \%)$ according to MCCS.

In a recent study by the Clinical Research Office of the Endourological Society, the data of 11,885 patients (1852 with only renal stones, 8676 with only ureteral stones, and 1145 patients with both types of stone), who underwent ureteroscopy in 114 centers in 32 countries, were prospectively examined [16]. The postoperative complication rate was reported as $3.5 \%$, and most of the cases that developed complications were Clavien grade 1 or $2(2.8 \%)$. Intraoperative complications were also reported separately, including bleeding (1.4\%), perforation $(1.0 \%)$, ureteral avulsion $(0.1 \%)$, and conversion to open surgery $(0.1 \%)$. The most frequent complication in that series was fever $(1.8 \%)$. Bleeding was reported at a rate of $0.4 \%$, and a blood transfusion was required in $0.2 \%$ of patients. In addition, sepsis developed in $38(0.3 \%)$ patients, and mortality in five patients for various other reasons. SCS is the most common classification system used in classifying intraoperative adverse events [9]. In our series, Satava grade 1 was seen in a total of 60 patients. However, there were six $(0.6 \%)$ patients with malfunctioning or breakage of instruments in the Satava 1 group. We consider that it is not appropriate to evaluate this as an intraoperative complication. This situation should be referred to as an intraoperative event, not as a complication. In addition, the most common intraoperative complication in our patients was minimal mucosal injury (3.7\%). However, grade 3 complications were not observed. The most common grade $2 \mathrm{~b}$ complication was requirement of repeat RIRS (5.6\%) after the stone could not be accessed for various reasons. Among the patients with grade $2 \mathrm{~b}$ complications, the surgery had to be terminated early in six $(0.6 \%)$ due to vision impairment caused by severe bleeding. Nevertheless, all of these bleedings were self-limited and did not require a blood transfusion or surgical intervention. While minimal mucosal erosions or tears (grade 1) can heal conservatively without a DJ stent, severe mucosal injuries, such as ureteral perforation (grade 2), are mostly treated by placing a DJ stent. In a study by Ural et al., mild mucosal injury was seen in only $10(4.3 \%)$ patients, and serious mucosal cleft was observed in three $(1.3 \%)$ patients and treated by inserting a urethral DJ stent [17]. In our cases, mild and severe mucosal injuries were rare, similar to the literature. Traxer et al. reported that preoperative ureter stenting reduced the risk of severe ureteric injury by 7 times [13]. In our study, no statistically significant relationship was found between preoperative stenting and absence of complications, and the relationship between preoperative DJ insertion and ureteral injury was not evaluated in our series. Although SCS includes the minor complications mentioned above, conditions such as fever and sepsis are not classified in this system. We consider that SCS evaluates whether the outcome of the operation is a success because it is based on whether the operation is to be completed then or postponed. We think that malfunctioning or breakage of instruments is not a complication, and therefore they should not be included in SCS.

Breda et al. reported the overall complication rate for RIRS as $8 \%$ and the major complication rate as $1.9 \%$ according to MCCS [18]. In a prospective randomized study by Sabnis et al. conducted with 35 patients that underwent RIRS, the rate of Clavien grade 1 complications was determined as $11.4 \%$, but no other complication was reported [20]. In our series, $76 \%$ of the patients that developed complications were classified as grade 1. Major complications were observed in $18(1.9 \%)$ patients. Two patients had a 
ureteral stricture $(0.2 \%)$, one had urosepsis $(0.1 \%)$, and a further two died due to urosepsis $(0.2 \%)$. Therate of postoperative fever resolved with an antipyretic was consistent with the literature in our study, but the rate of fever requiring antibiotic replacement (grade 2) was lower in our study [21]. In a study by Tian et al., the rate of fever was found to be $17.5 \%$, and diabetes mellitus, elevated preoperative C-reactive protein (CRP), high stone burden, positive stone culture, and positive renal pelvis culture were shown to be among the reasons that could cause fever [22]. In another study, Yong et al. showed that operative time was an important factor for postoperative fever [21]. In our series, operative time was longer in the complication group. In the subgroup analysis performed, operation success was found to be the factor affecting major (>grade 3) complication. We consider that factors that affect the success of the operation, such as stone size and density, also indirectly affect the probability of major complications.

In the literature, postoperative hematuria has been reported at a rate of 5-7.1\%, and it has been emphasized that the use of stone burden, a high CT attenuation value, or use of UAS increased the incidence of postoperative hematuria $[21,23]$. In a study conducted by Shah et al., it was stated that postoperative hematuria was common in patients with stones of high $\mathrm{CT}$ attenuation, which was caused by increased mucosal damage as a result of more effort being required to fragment the stone [24]. In our study, postoperative hematuria was the second most common complication with a rate of $3.6 \%$ and was found be related to increased stone burden, presence of residual stone, and operative time while there was no relationship between the use of UAS and postoperative hematuria development. Although postoperative hematuria is more common in patients with low CT attenuation values, it is evaluated as borderline non-significant. This situation can be explained by the prolongation of operative time as a result of the decrease in the detection of stones with low $\mathrm{HU}$ values on fluoroscopy. This idea is supported by the statistically significant negative correlation between $\mathrm{HU}$ and operative time and the significant relationship between operative time and postoperative hematuria development.

Although other complications are extremely rare, they can lead to serious situations if they occur. PostRIRS sepsis is one of the most serious complications [25]. In a retrospective study by Fan et al., sepsis was seen in two (0.88) patients after RIRS, and the infective complication rates were found to vary between 1.7 and $18.8 \%$ [26]. Berardinelli et al. reported the rate of sepsis to be $0.7 \%$ [27]. In our series, sepsis was observed in three $(0.3 \%)$ patients, which is consistent with the literature. In studies evaluating factors related to the development of infective complications, pyuria, operative time, infection stones, diabetes mellitus, elevated preoperative CRP, high stone burden, and positive stone culture were found as independent predictive factors $[22,26]$. In addition, another major complication, steinstrasse, was observed at a rate of $1.9 \%$ in aprevious study [21]. In our study, non-obstructive steinstrasse was detected at a rate of $0.6 \%$ and obstructive steinstrasse at $0.5 \%$. Ural et al. determined that the rate of major complications according to SCS was $8.9 \%$ [17]. Abnormal renal anatomy and presence of multiple stones each increased complication development by 2.7 times while the stone-free status decreased complication development by 4.2 times. According to MCCS, stone-free status was determined as an independent predictive factor in the development of major complications. In addition, according to SCS, stone size, stone density, and fluoroscopy time were independent risk factors in predicting the requirement of endoscopic, open or laparoscopic treatment.

The main limitations of our study are that it had a retrospective design and the operation was performed by specialists with different surgical experience. Another limitation is that we did not examine factors that had been previously shown to be associated with the development of complications, such as CRP, stone culture, and stone type, nor did we evaluate late-term complications.

\section{Conclusion}

RIRS is an effective and safe surgical method in the treatment of renal stones. In our study, the presence of abnormal kidney anatomy, multiple stones, operative time, and residual stones were determined as independent predictive factors for complication development after RIRS whereas the presence of residual stones was the only independent predictive factor for major complications. MCCS and SCS are fast and easily applicable classification systems for grading RIRS-related complications. 


\section{Reference}

1. Abe R, Etori K, Kato T, Sato K, Nihon Hinyokika Gakkai Zasshi (1990) Experience with transurethral uretero-nephrolithotripsy using flexible nephro-ureteroscope.1667-1674, Cilt 81.

2. Puppo P, Bottino P, Germinale F, Caviglia C, Ricciotti G, Giuliani L (1990) F lexible antegrade and retrograde nephroscopy: review of 50 cases. Eur Urol, 17:193-199.

3. Türk C, Neisius A, Petrik A, Seitz C, Skolarikos A, Thomas K (2020) Guidelines on urolithiasis. European Association of Urology.

https://uroweb.org/guideline/urolithiasis/2020

4. Berardinelli F, Proietti S, Cindolo L et al. (2016) A prospective multicenter European study on flexible ureterorenoscopy for the management of renal stone. Int Braz J Urol, 42: 479-486.

5. Gunnar Wendt-Nordahl, Tuna Mut, Patrick Krombach, Maurice Stephan Michel, Thomas Knoll. (2011) Do new generation flexible ureterorenoscopes offer a higher treatment success than their predecessors? Urol Res, 39(3):185-8.

6. Robert Geraghty, Omar Abourmarzouk, Bhavan Rai, Chandra Shakhar Biyani, Nicholas J Rukin, Bhaskar K Somani. (2015) Evidence for Ureterorenoscopy and Laser Fragmentation (URSL) for Large Renal Stones in the Modern Era. Curr Urol Rep, 16(8):54.

7. Geavlete P, Georgescu D, Niţă G, Mirciulescu V, Cauni V (2006) Complications of 2,735 retrograde semirigid ureteroscopy procedures: a single-center experience. J Endourol, 20:179-185.

8. Mandal S, Goel A, Singh MK et al. (2012) Clavien Classification of Semirigid Ureteroscopy Complications: A Prospective Study. Urology, 80(5):995-1001

9. Satava, RM. (2005) İdentification and reduction of surgical error using simulation. Minim Invasive Ther Technol, Cilt 14(4):257-261

10. Dindo D, Demartines N, Clavien PA. (2004) Classification of surgical complications: a new proposal with evaluation in a cohort of 6336 patients and results of a survey. Ann Surg, Cilt 2, 240:205-213.

11. Lin KJ, Lin PH, Chu SH, Chen HW, Wang TM, Chiang YJ et al. (2014) The impact of climate factors on the prevalence of urolithiasis in Northern Taiwan. Biomed J, 37:24-30.

12. Ramon de Fata F, Garcia-Tello A, Andres G, Redondo C, Meilan E, Gimbernat H et al. (2014) Comparative study of retrograde intrarenal surgery and micropercutaneous nephrolithotomy in the treatment of intermediate-sized kidney stones. Actas Urol Esp, 38:576-583.

13. Traxer O, Thomas A. (2013) Prospective evaluation and classification of ureteral wall injuries resulting from insertion of a ureteral access sheath during retrograde intrarenal surgery. J Urol ,189:580-584.

14. Resorlu B, Unsal A, Gulec H, Oztuna D. (2012) A new scoring system for predicting stone-free rate after retrograde intrarenal surgery: the 'ResorluUnsal stone score'. Urology, 80:512-518.

15. Schoenthaler M, Wilhelm K, Katzenwadel A, Ardelt P, Wetterauer U, Traxer O, Miernik A. (2012) Retrograde intrarenal surgery in treatment of nephrolithiasis: is a $100 \%$ stone-free rate achievable? J Endourol, 26:489-493.

16. DelaRosette J, Denstedt J, Geavlete P, Keeley F, Matsuda T, Pearle M, Preminger G, Traxer O, Group CUS. (2014) The clinical research office of the endourological society ureteroscopy global study: indications, complications, and outcomes in 11,885 patients. J Endourol, 28:131-139.

17. Oguz U, Resorlu B, Ozyuvali E, Bozkurt OF, Senocak C, Unsal A. (2014) Categorizing intraoperative complications of retrograde intrarenal surgery. Urol Int, 92(2):164-168. 
18. Breda A, Angerri O (2014) Retrograde intrarenal surgery for kidney stones larger than $2.5 \mathrm{~cm}$. Curr Opin Urol, 24:179-183.

19. Karakoyunlu N, Ekici M, Yesil S, Zengin K, Goktug G, Ozok U. (2014) Comparison of complications associated with standard and totally tubeless percutaneous nephrolithotomy according to modified Clavien grading: a multicenter retrospective study. Kaohsiung J Med Sci, 30:613-618.

20. Sabnis RB, Ganesamoni R, Doshi A, Ganpule AP, Jagtap J, Desai MR. (2013) Micropercutaneous nephrolithotomy (microperc) vs retrograde intrarenal surgery for the management of small renal calculi: a randomized controlled trial. BJU Int,112:355-361.

21. Guangjun Duan, Yong Xu· Zhiqian Min. Shaw P. Wan. Haibo Nie·. (2018) Complications of retrograde intrarenal surgery classified by the modified Clavien grading system. Urolithiasis, 46:197-202.

22. Tian Lİ, Xiang-Zhou Sun, De-Hui Lai, Xu Li, Yong-Zhong He.(2018) Fever and Systemic İnflammatory Response Syndrome After Retrograde İntrarenal Surgery: Risk Factors and Predictive Model.Kaohsiung J Med, 34(7):400-408.

23. Tae Jin K, In Jae, Jung Keun L, Hak Min L, Chang Wook J, Sung Kyu H, Seok-Soo B, Jong Jin Oh. (2019) Analysis of factors affecting re-admission after retrograde intrarenal surgery for renal stone. World J Urol, 37(6):1205-1210.

24. Shah K, Kurien A, Mishra S, Ganpule A, Muthu V, Sabnis RB. (2010) Predicting effectiveness of extracorporeal shockwave lithotripsy by stone attenuation value. J Endourol, 24:1169-1173.

25. Shoshany O, Margel D, Finz C. (2015) Percutaneous nephrolithotomy for infection stones: what is the risk for postoperative sepsis? A retrospective cohort study. Urolithiasis, 43:237-242.

26. Fan S, Gong B, Zet Hao et al. (2015) Risk factors of infectious complications following flexible ureteroscope with a holmium laser: a retrospective study. Int J Clin Exp Med, 8(7):11252-11259.

27. Francesco Berardinelli, Piergustavo De Francesco, Michele Marchioni, Nicoletta Cera, Silvia Proietti, Derek Hennessey, Orietta Dalpiaz, Cecilia Cracco, Cesare Scoffone, Luigi Schips, Guido Giusti, Luca Cindolo. (2016) Infective complications after retrograde intrarenal surgery: a new standardized classification system. Int Urol Nephrol, 48(11):1757-1762

\section{Hosted file}

Table-1.pdf available at https://authorea.com/users/381093/articles/513845-factors-affectingintraoperative-and-postoperative-complications-of-rirs-classified-by-the-clavien-andsatava-grading-systems

\section{Hosted file}

Table-2.pdf available at https : //authorea.com/users/381093/articles/513845-factors-affectingintraoperative-and-postoperative-complications-of-rirs-classified-by-the-clavien-andsatava-grading-systems

\section{Hosted file}

Table-3.pdf available at https : //authorea.com/users/381093/articles/513845-factors-affectingintraoperative-and-postoperative-complications-of-rirs-classified-by-the-clavien-andsatava-grading-systems

\section{Hosted file}

Table-4.pdf available at https : //authorea.com/users/381093/articles/513845-factors-affectingintraoperative-and-postoperative-complications-of-rirs-classified-by-the-clavien-andsatava-grading-systems 